\title{
Carbon to Oxygen Abundance Ratios in the Atmospheres of Carbon Stars of the Orion and Perseus Galactic Arms
}

\author{
I. EGLITIS and M. EGLITE \\ Radioastrophysical Observatory, Riga, Latvia
}

The abundance ratio $\mathrm{C} / \mathrm{O}$ in the atmospheres of carbon stars of the Orion and Perseus galactic arms is determined on the basis of a great number of homogeneous spectra obtained with the 2.6-m telescope of the Byurakan Astrophysical Observatory (Armenia) in 1990-1991. The reciprocal dispersion for observations of faint carbon stars was $101-136 \AA / \mathrm{mm}$ and the wavelength range was 4000-6800 $\AA$. We used the empirically-obtained correlation between the intensities of the CN Red System and $\mathrm{C}_{2}$ Swan bands, on the one hand, and $\mathrm{C} / \mathrm{O}$ ratios determined from model calculations of synthetic spectra (which were compared to 30 high-resolution spectra of carbon stars [Lambert et al. 1987, ApJS, 62, 273]), on the other hand, and so obtained the individual $\mathrm{C} / \mathrm{O}$ abundances in the atmospheres of 342 faint carbon stars. Twenty-eight high-carbon-abundance $\mathrm{C}$ stars were found. They all have similar spatial distribution and many common spectral pecularities.

The distributions of carbon stars by $\mathrm{C} / \mathrm{O}$ abundance ratio interval were studied in the Orion and Perseus galactic arms. In Orion most carbon-star atmospheres have carbon to oxygen ratios in the interval $1.0 \leq \mathrm{C} / \mathrm{O} \leq 1.1$, but in the Perseus Arm most are in the interval $1.1 \leq \mathrm{C} / \mathrm{O} \leq 1.2$.

A detailed discussion of these results has been submitted to Astrophysics and Space Science. 